\title{
Circular RNA and gene expression profiles in gastric cancer based on microarray chip technology
}

\author{
WEIGUO SUI $^{1 *}$, ZHOUFANG SHI $^{2 *}$, WEN XUE $^{1}$, MINGLIN OU $^{1}$, YING ZHU ${ }^{1}$,

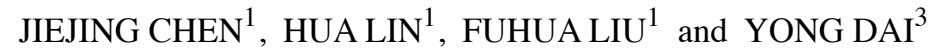 \\ ${ }^{1}$ Nephrology Department of Guilin No. 181 Hospital, Guangxi Key Laboratory of Metabolic Diseases Research, \\ Guilin, Guangxi 541004; ${ }^{2}$ College of Life Science of Guangxi Normal University, Guilin, Guangxi 541004; \\ ${ }^{3}$ Clinical Medical Research Center, The Second Clinical Medical, College of Jinan University, \\ Shenzhen People's Hospital, Shenzhen, Guangdong 518020, P.R. China
}

Received August 25, 2016; Accepted January 9, 2017

DOI: 10.3892/or.2017.5415

\begin{abstract}
The aim of the present study was to screen gastric cancer (GC) tissue and adjacent tissue for differences in mRNA and circular (circRNA) expression, to analyze the differences in circRNA and mRNA expression, and to investigate the circRNA expression in gastric carcinoma and its mechanism. circRNA and mRNA differential expression profiles generated using Agilent microarray technology were analyzed in the GC tissues and adjacent tissues. qRT-PCR was used to verify the differential expression of circRNAs and mRNAs according to the interactions between circRNAs and miRNAs as well as the possible existence of miRNA and mRNA interactions. We found that: i) the circRNA expression profile revealed 1,285 significant differences in circRNA expression, with circRNA expression downregulated in 594 samples and upregulated in 691 samples via interactions with miRNAs. The qRT-PCR validation experiments showed that hsa_circRNA_400071, hsa_circRNA_000543 and hsa_circRNA_001959 expression was consistent with the microarray analysis results. ii) 29,112 genes were found in the GC tissues and adjacent tissues, including 5,460 differentially expressed genes. Among them, 2,390 differentially expressed genes were upregulated and 3,070 genes were downregulated. Gene Ontology (GO) analysis of the differentially expressed genes revealed these genes involved in biological process classification, cellular component classification and molecular function classification. Pathway analysis of the differentially expressed genes
\end{abstract}

Correspondence to: Professor Yong Dai, Clinical Medical Research Center, The Second Clinical Medical, College of Jinan University, Shenzhen People's Hospital, No. 1017 East Road, Shenzhen, Guangdong 518020, P.R. China

E-mail: daiyong22@aliyun.com

*Contributed equally

Key words: gastric cancer, circRNA, gene expression profile, biomarker, differential expression, diagnosis identified 83 significantly enriched genes, including 28 upregulated genes and 55 downregulated genes. iii) 69 differentially expressed circRNAs were found that might adsorb specific miRNAs to regulate the expression of their target gene mRNAs. The conclusions are: i) differentially expressed circRNAs had corresponding miRNA binding sites. These circRNAs regulated the expression of target genes through interactions with miRNAs and might become new molecular biomarkers for GC in the future. ii) Differentially expressed genes may be involved in the occurrence of GC via a variety of mechanisms. iii) CD44, CXXC5, MYH9, MALAT1 and other genes may have important implications for the occurrence and development of GC through the regulation, interaction, and mutual influence of circRNA-miRNA-mRNA via different mechanisms.

\section{Introduction}

Gastric cancer (GC) is a malignant tumor that occurs in the gastric mucosa of the digestive tract (1). GC has become the fourth most common cancer in the world and ranks second as the cause of cancer death (2). GC is one of the main diseases in the clinic, with features such as a high incidence rate, a lack of obvious clinical symptoms, invasion and metastasis and a low cure rate (3).

Surgical resection is an ideal method for the treatment of gastric cancer. A total of $90 \%$ of early gastric cancer patients survive 5 years after the surgical resection of the lesions (4). However, most patients confirmed with gastric cancer are in the progressive stage due to the lack of specific symptoms of early gastric cancer (5). Therefore, the mortality rate of gastric cancer stays at a high level. Although the cancer genes, tumor suppressor genes and tumor signaling pathways associated with gastric cancer have been discovered and confirmed, its pathogenesis is not fully clear. Thus, the search for new biological targets to monitor and intervene in GC carcinogenesis is urgent for $\mathrm{GC}$ prevention.

Circular RNA (circRNA) is a class of endogenous non-coding RNA (ncRNA) molecules that regulate gene expression (6). Some circRNAs can act as microRNA (miRNA) sponges to regulate the expression levels of other 
related RNAs (7,8). In other words, circRNAs can use miRNA response elements to bind miRNAs and block the inhibitory effects of miRNAs on the expression of their target genes (9).

circRNAs play very important roles in many diseases, such as nervous system disorders, atherosclerosis, diabetes and cancer (10). Although the role of circRNAs in the occurrence of the disease has received attention, research into the relationship between circRNAs and gastric cancer is rare.

In the present study, we analyzed the differential expression profiles of circRNAs in gastric cancer tissues and adjacent tissues to explore the relationship between the circRNA expression and GC and to provide a preliminary theoretical basis for search of biomarkers for the early diagnosis and malignant progression of GC.

circRNAs have important functions within living organisms. At present, circRNAs are known to function through a number of other molecules, such as splicing factors, RNA polymerase II (11), nuclear small ribose nucleoprotein (snRNP) (12), and miRNAs (13), to regulate host gene (host gene) linear mRNA production; these interactions can promote or inhibit the transcription of the corresponding linear mRNA. If the molecular mechanism is based on the circRNA regulation of host genes as described above, we can analyze the same gene produced by circRNA and linear mRNA in the tested sample to evaluate trends in changes of expression to speculate on the molecular mechanism underlying the functions of circRNAs in the organism.

\section{Materials and methods}

Human subjects: Patients and healthy controls. The 8 GC tissues and adjacent tissue samples used in this study were collected from the Shenzhen People's Hospital. All patients aged from 42 to 76 years who had not been treated with radiotherapy or chemotherapy prior to surgery were included. Specimens were collected from March to May in 2015. The experimental study was approved by the hospital ethics committee. The present study obtained the consent of the patients or their families by signing the informed consent forms.

Tissue sample collection. GC and adjacent tissues $\left(1 \mathrm{~cm}^{3}\right)$ obtained after stomach cancer surgeries were immediately washed with $0.9 \% \mathrm{NaCl}$ (RNase-free) and quickly dipped in RNase inhibitor (Epicentre, Madison, WI, USA) according to the manufacturer's instructions. After storage at $4^{\circ} \mathrm{C}$ overnight, the depressors were removed from the biopsies and the biopsies were stored at $-80^{\circ} \mathrm{C}$ for further testing.

RNA sample preparation. Total RNA was extracted from the renal cortex pieces using TRIzol (Invitrogen) according to the manufacturer's instructions. The concentration and quality of the total RNA were measured by UV absorbance at $260 \mathrm{~nm}$ and $280 \mathrm{~nm}(\mathrm{~A} 260 / 280)$ and verified by gel electrophoresis.

Screening and identification of gastric cancer-associated circRNAs. Sample labeling and array hybridization were performed according to the manufacturer's protocol (Arraystar Inc., Rockville, MD, USA). Briefly, total RNA was digested with RNase R (Epicentre) to remove linear RNAs and to enrich circular RNAs. Then, the enriched circular RNAs were amplified and transcribed into fluorescent cRNA using a random priming method (Arraystar Super RNA Labeling kit; Arraystar). The labeled cRNAs were purified with the RNeasy Mini kit (Qiagen). The concentration and specific activity of the labeled cRNAs (pmol Cy3/ $\mu \mathrm{g}$ cRNA) were measured by NanoDrop ND-1000. A total of $1 \mu \mathrm{g}$ of each labeled cRNA was fragmented by adding $5 \mu \mathrm{l}$ of 10X blocking agent and $1 \mu \mathrm{l}$ of $25 \mathrm{X}$ fragmentation buffer. Then, the mixtures were heated at $60^{\circ} \mathrm{C}$ for $30 \mathrm{~min}$. Finally, $25 \mu \mathrm{l}$ of $2 \mathrm{X}$ hybridization buffer was added to dilute the labeled cRNA. A total of $50 \mu \mathrm{l}$ of the hybridization solution was dispensed into the gasket slide and assembled to construct the circRNA expression microarray slide. The slides were incubated for $17 \mathrm{~h}$ at $65^{\circ} \mathrm{C}$ in an Agilent Hybridization Oven. The hybridized arrays were washed, fixed and scanned using the Agilent scanner G2505C.

Screening and identification of gastric cancer-associated $m R N A s$. Human whole genome oligonucleotide microarrays representing all known genes and transcripts in the human gene were applied in the extensive view. The sequences were collected from a wide range of sources and were validated and optimized through comparison to the assembly of the human genome. Sample labeling and hybridization were performed in accordance with the Agilent One-Color Microarray-Based Gene Expression Analysis experiment scheme with slight modifications. First, the total RNA was amplified from each sample and used as a Cy3-UTP marker. Second, the labeled cRNAs were purified with the RNeasy Mini kit and the concentration and activity were detected with the NanoDrop ND-1000. Finally, the slides were incubated, washed, fixed and scanned using the Agilent scanner G2505C.

Differentially expressed gene analysis and functional analysis. We used the Agilent Feature Extraction v11.0.1.1 software graph chip to read the values and to obtain the original data. The GeneSpring GX v12.1 software was used for quantile normalization and subsequent data processing of the original data. After the original data standardization, we screened for the high quality probe (a probe that is marked as 1 for detection in at least two samples). Two groups of samples with significantly differentially expressed genes were identified by scatter plot screening. The difference between the gene expression in the two samples was validated through fold change screening. Hierarchical clustering was performed using a script prepared by Kang Cheng (Shanghai, China). The GO analysis and pathway analysis were performed using the standard enrichment calculation method.

Data acquisition and analysis. Scanned images were imported into the Agilent Feature Extraction software for raw data extraction. Quantile normalization of the raw data and subsequent data processing were performed using the $\mathrm{R}$ software package. When comparing two groups of profile differences (i.e., disease vs. control), the 'fold change' (i.e., the ratio of the group averages) was computed between the groups for each circRNA. The statistical significance of the difference may be conveniently estimated using the t-test. circRNAs with fold changes $<1.5$ and P-values $<0.05$ were selected as significantly differentially expressed genes. 
Table I. Real-time qRT-PCR primer list.

\begin{tabular}{|c|c|c|c|}
\hline circRNA & Primer sequences $\left(5^{\prime}-3^{\prime}\right)$ & Product sizes/bp & Annealing temperature $/{ }^{\circ} \mathrm{C}$ \\
\hline \multirow[t]{2}{*}{ GAPDH(HUMAN) } & F: GGGAAACTGTGGCGTGAT & & \\
\hline & R: GAGTGGGTGTCGCTGTTGA & 299 & \\
\hline \multirow[t]{2}{*}{ hsa_circRNA_400071 } & F: CCCAAGGGTTTGGTGGGAT & & \\
\hline & R: AGAGCCCAGAGTGGGAGAAGTC & 109 & \\
\hline \multirow[t]{2}{*}{ hsa_circRNA_000792 } & F: AGGAAGTACAATGAAGTGCCAGT & & \\
\hline & R: AGAATGCCCAAAGACAAAGC & 157 & \\
\hline \multirow[t]{2}{*}{ hsa_circRNA_000543 } & F: GAATAGATTTCAGCTTTATGC & & \\
\hline & R: CATAACTGATCTGACTTTGTATG & 83 & 60 \\
\hline \multirow[t]{2}{*}{ hsa_circRNA_001959 } & F: GTCTAGTGGAGCAGGTGGAGG & & \\
\hline & R: AGGGCCATGACCTGGGTTA & 137 & \\
\hline \multirow[t]{2}{*}{ hsa_circRNA_400066 } & F: GACCCTTCCTGGCGGTTAC & & \\
\hline & R: AGACCCACAAGCCCATTCC & 237 & \\
\hline \multirow[t]{2}{*}{ hsa_circRNA_001066 } & F: CTTTTTCAGACTATTTCGTGTCC & & \\
\hline & R: CTTCTGGAAGTACCCAATATGC & 88 & \\
\hline
\end{tabular}

F, forward; R, reverse.

Validation of differentially expressed circRNAs by qRT-PCR. We randomly chose circRNAs with upregulated and downregulated expression to verify the results using qRT-PCR. The primers are listed in Table I. The cycle parameters for the PCR reaction were $95^{\circ} \mathrm{C}$ for $5 \mathrm{~min}$, followed by 40 cycles of a denaturing step at $95^{\circ} \mathrm{C}$ for $10 \mathrm{sec}$ and an annealing/extension step at $60^{\circ} \mathrm{C}$ for $60 \mathrm{sec}$. All reactions were run in triplicate. The Rotor-Gene Real-Time Analysis software 6.0 was used for the data analysis.

The fold changes were calculated with the $2^{-\Delta \Delta \mathrm{Ct}}$ method (14). To reduce errors caused by the RNA concentration and transcription efficiency, we used the housekeeping gene GAPDH (its expression level was basically constant in the different samples) as an internal control. The relative gene contents under the test conditions were the gene value ratios of the sample and internal control.

Correlation analysis of circRNAs and mRNAs in gastric cancer. We used the Agilent circRNA and mRNA expression profile microarray data to screen the differentially expressed circRNA and mRNA gene expression profiling information for the integration expression analysis. To investigate whether differentially expressed circRNAs could perform miRNA-specific adsorption, we evaluated the regulation of the expression of their target gene mRNAs. We investigated the functions of circRNAs and the mechanism underlying the occurrence of gastric cancer.

\section{Results}

Screening of the differentially expressed circRNA. The circRNA expression profile in the GC and adjacent tissues is shown in Fig. 1. Through the data analysis, we selected 4,458 circRNAs whose expression levels were upregulated or downregulated in the GC tissues.

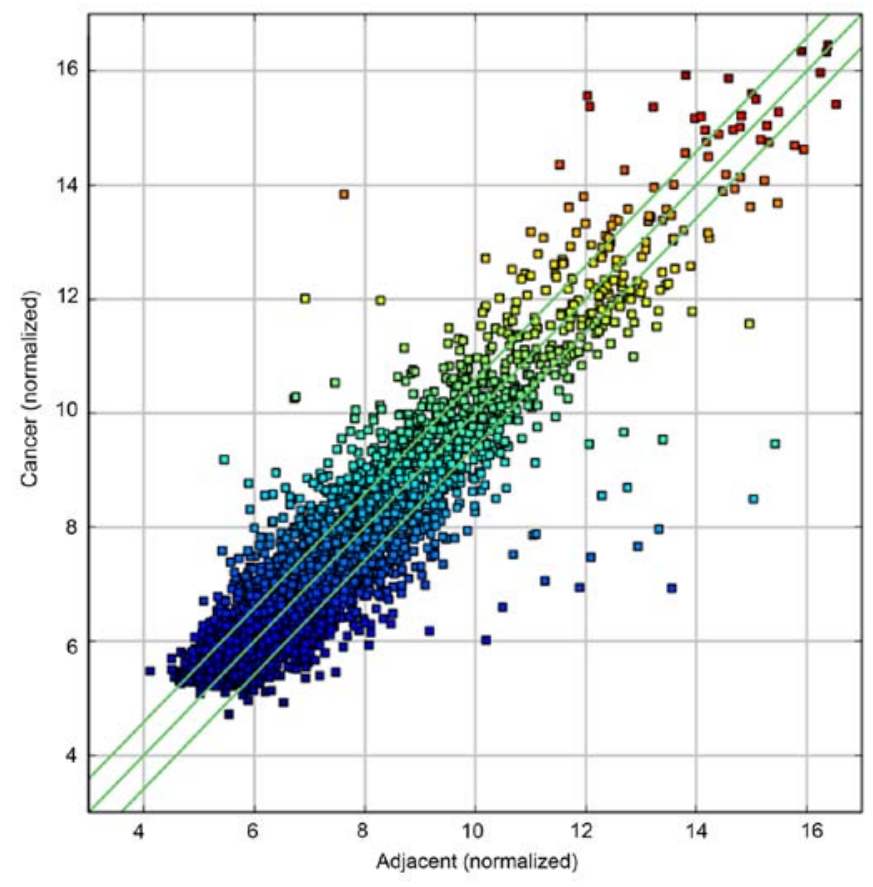

Figure 1. Scatter plot of differentially expressed circRNAs.

To determine whether there was a significant difference in circRNA expression between the two groups of samples, we performed a statistical analysis of data from the screened differentially expressed circRNAs. The circRNA expression levels were normalized to the same order of magnitude prior to the statistical analysis. circRNAs whose differences in expression were $>2$-fold and had P-values $<0.05$ were regarded as differentially expressed circRNAs. There were 467 differentially expressed circRNAs, of which 214 were significantly upregulated and 253 were significantly downregulated. 
A1
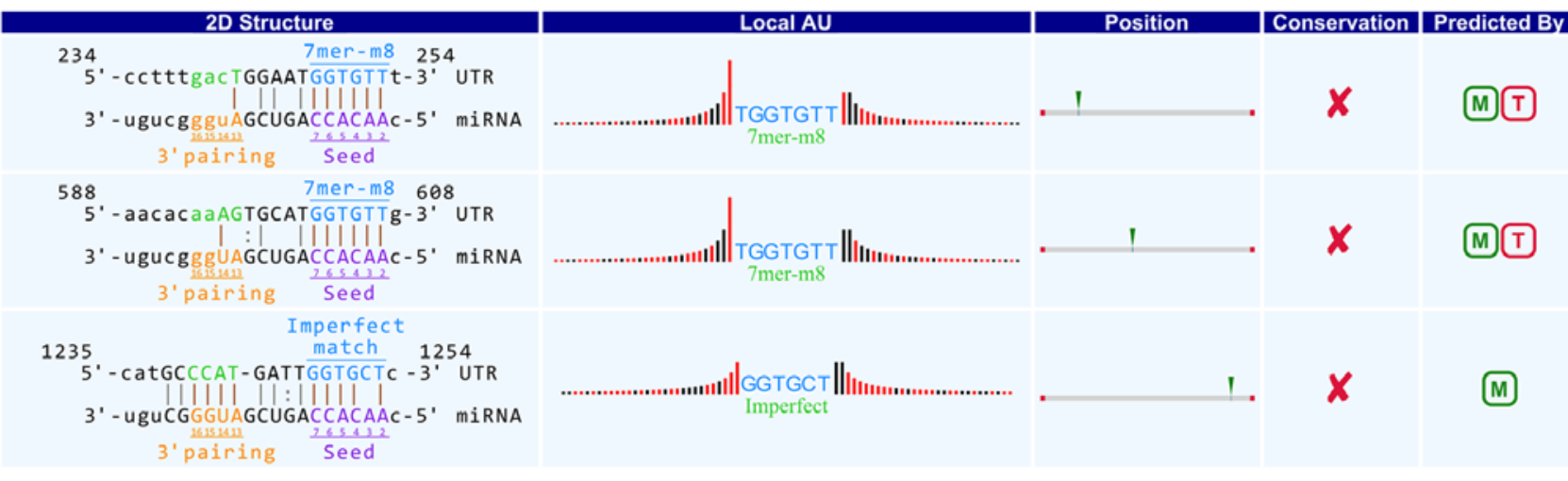

A2
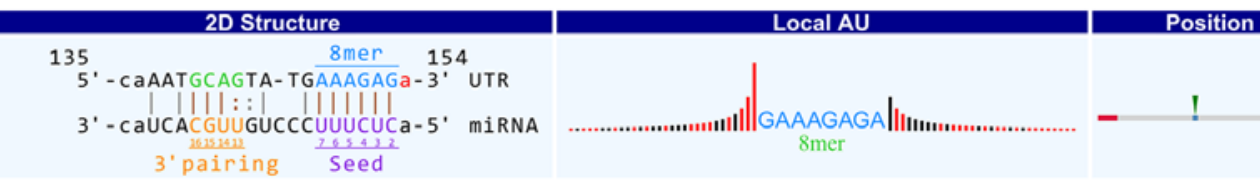

Conservation Predicted By

B1
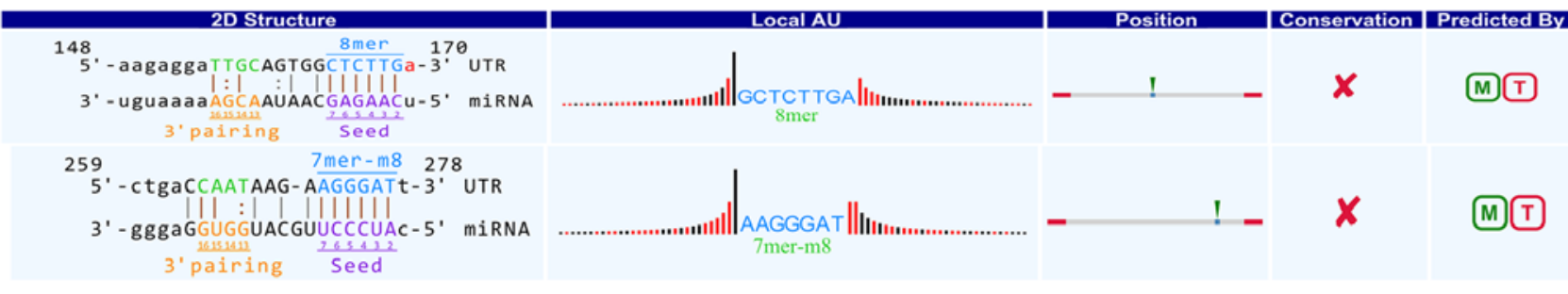

B2
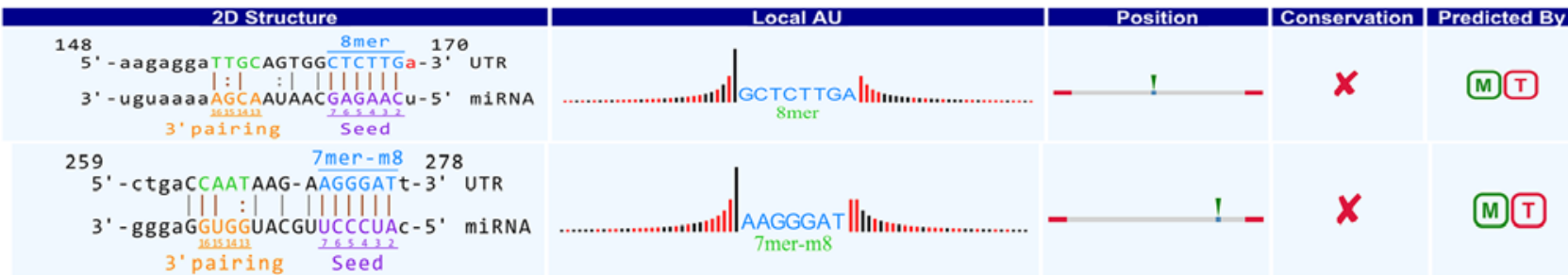

Figure 2. The detailed annotation for circRNA-miRNA interactions (A1, complementary situations of hsa_circRNA_101242 and miR-21-3p; A2, complementary situations of hsa_circRNA_001622 and miR-130b-5p; B1, complementary situations of hsa_circRNA_103122 and miR-135a-5p; B2, complementary situations of hsa_circRNA_101320 and miR-335-5p).

$q R T-P C R$ validation results. After the comparison and the analysis of the differentially expressed multiple circRNAs in the microarray experiments, we randomly verified three circRNAs (hsa_circRNA_400071, hsa_circRNA_000792 and hsa_circRNA_000543) with upregulated expression and three circRNAs (hsa_circRNA_001959, hsa_circRNA_400066 and hsa_circRNA_001066) with downregulated expression.

Using GAPDHA as the internal control, we used qRT-PCR to verify the expression levels of the above 6 circRNAs. The results are shown in Table II. There were significant differences in all of the selected circRNAs $(\mathrm{P}<0.05)$ with the exception of hsa_circRNA_000543 $(\mathrm{P}=0.235)$. The objective circRNA validation rate was $5 / 6$, showing that the circRNA expression profiles were reliable.

Interaction between differentially expressed circRNAs and miRNAs. Differentially expressed circRNAs contained corresponding miRNA binding sites. To facilitate the investigation, the interactions between miRNAs and circRNAs were predicted by an in-house miRNA target prediction software (Arraystar). The circRNA/miRNA interaction infor-
Table II. Verification of the circRNA microarray results.

\begin{tabular}{lccl}
\hline circRNA & Fold change & $2^{-\Delta \Delta \mathrm{Ct}}$ & P-value \\
\hline hsa_circRNA_400071 & 3.77 & 3.09 & 0.007 \\
hsa_circRNA_000792 & 3.63 & 1.28 & 0.046 \\
hsa_circRNA_000543 & 3.62 & 1.16 & 0.235 \\
hsa_circRNA_001959 & -2.14 & 0.29 & 0.00005 \\
hsa_circRNA_400066 & -2.33 & 1.27 & 0.025 \\
hsa_circRNA_001066 & -4.04 & 0.67 & 0.002 \\
\hline
\end{tabular}

mation for all circRNAs is noted in detail. For example, in hsa_circRNA_101242, the 248th-253rd and the 602nd-607th nucleotides starting from the 5 ' terminus in the high-expression gene PAN3 were completely complementary to the miR-21-3p seed region in the 7 mer-m 8 binding mode. However, the 248th-253rd nucleotides starting from the 5 ' terminus were not completely complementary to the miR-21-3p seed region (Fig. 2A1). 


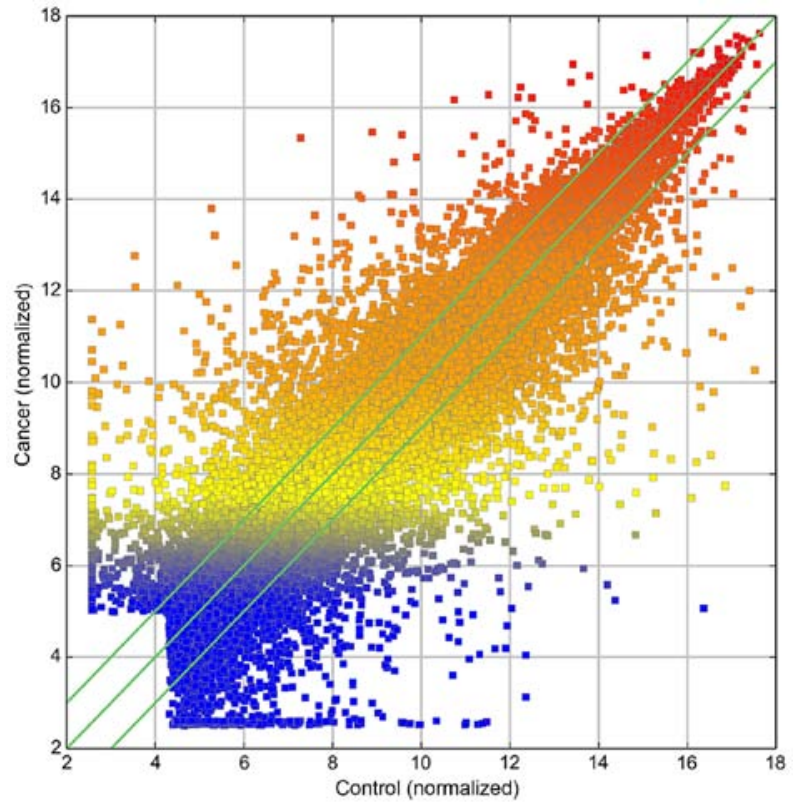

Figure 3. Scatter plot of the differentially expressed genes.

In hsa_circRNA_001622, the 148th-153rd nucleotides starting from the $5^{\prime}$ terminus in the high-expression gene SLAMF6 gene were completely complementary to the miR-130b-5p seed region in the 8mer binding mode (Fig. 2A2). Genes including PPP4R1, PPP4R1, TMCO3, SNX13 and LPAR1 were complementary to the miR-130b-5p seed region.

In hsa_circRNA_103122, the 609th-614th nucleotides starting from the 5 terminus in the low-expression gene DONSON were completely complementary to the miR-135a-5p seed region in the Offset 6 mer binding mode (Fig. 2B1). Genes including MYH14, ATG3, PDS5A, MYO10 and STXBP5 were complementary to the miR-135a-5p seed region.

In hsa_circRNA_101320, the 164th-169th nucleotides starting from the $5^{\prime}$ terminus in the low-expression gene PRMT5 were completely complementary to the miR-335-5p seed region in the 8 mer binding mode. Moreover, the 164th-169th nucleotides starting from the 5 ' terminus in the low-expression gene PRMT5 were completely complementary to the miR-188-5p seed region in the 7mer-m8 binding mode (Fig. 2B2).

mRNA GeneChip analysis. miRNA expression profiles were constructed in gastric cancer tissues and adjacent tissues. The data were analyzed and 29,112 genes were found in the gastric cancer tissues and adjacent tissues as shown in Fig. 3.

Genes with known expression levels were included in the two groups of sample statistics. To determine whether there was a significant difference in expression between the two groups of samples, gene expression was normalized to the same order of magnitude in the two groups based on the mRNA expression profiles. The statistical analysis of the two groups of samples included fold differences $>2$-fold and $\mathrm{P}<0.05$, with visual differences between gene expression. Differential expression was detected for 5,460 genes in the two groups of samples. The differential gene expression was significantly upregulated in 239 genes, with SI showing the maximum difference in expression. Conversely, differential
Table III. The verification results of differential gene expression.

\begin{tabular}{lccl}
\hline Gene & Differential expression & $2^{-\Delta \Delta C t}$ & P-value \\
\hline MYH9 & -1.15 & 2.42 & 0.0001 \\
CD44 & 1.70 & 2.38 & 0.0002 \\
MALAT1 & -1.31 & 1.95 & 0.0005 \\
CXXC5 & 3.71 & 1.89 & 0.00008 \\
ARVCF & 4.25 & 0.85 & 0.041 \\
ARIH1 & -1.08 & 1.07 & 0.033 \\
\hline
\end{tabular}

gene expression was significantly downregulated in 3,070 genes, with ATP4B showing the greatest difference in gene expression.

$q R T-P C R$ validation results. Through access to the relevant literature combined with the analysis of the microarray experiments in the differentially expressed gene multiple alignment, we selected relative literature that reported microarray gene results for verification. Our analysis included three upregulated genes (MYH9, CD44 and MALAT1) and three downregulated genes (CXXC5, ARVCF and ARIH1) compared to GAPDHA for reference. The expression results of the six genes were analyzed by qRT-PCR for chip verification. The results were as follows: CD44, CXXC5 and ARVCF in the experimental group were upregulated by 2.38-, 1.89- and 0.82-fold, respectively, whereas MYH9, MALAT1 and ARIH1 in the experimental group were downregulated by $2.42-, 1.95$ and 1.07-fold, respectively, compared to the control group. The differences were significant $(\mathrm{P}<0.05)$. Target gene expression in the microarray analysis results was consistent with the confirmed mRNA expression levels from the microarray gene test results, indicating that the results were credible (Table III).

Differential gene bioinformatics analysis. GO analysis is the international standard gene function classification system. GO is used to describe biological genes and perform expression analysis of product attributes. GO includes three areas (biological process, cell component and molecular function). GO classifications are calculated for selected genes using a particular branch of the hypergeometric distribution relationship for each gene to obtain a P-value (under normal circumstances, the function of $\mathrm{P}<0.05$ for enrichment items). Genetic differences in the GO terms in the analysis of the experimental results indicate enrichment. GO suggests a role based on the differences in the gene analysis and can find enriched genes that are differentially categorized entries based on changes in different samples. Different genes may have related functions, such as those associated with signal transduction and positioning.

Differentially expressed genes were identified in the biological process (1,638 genes, including 841 upregulated genes and 797 downregulated genes), cell component (205 genes, including 119 upregulated genes and 86 downregulated genes) and molecular function categories (147 genes, including 155 upregulated genes and 92 downregulated genes) in the GO analysis. The GO analysis results of the differentially expressed genes are shown in Figs. 4-9. 


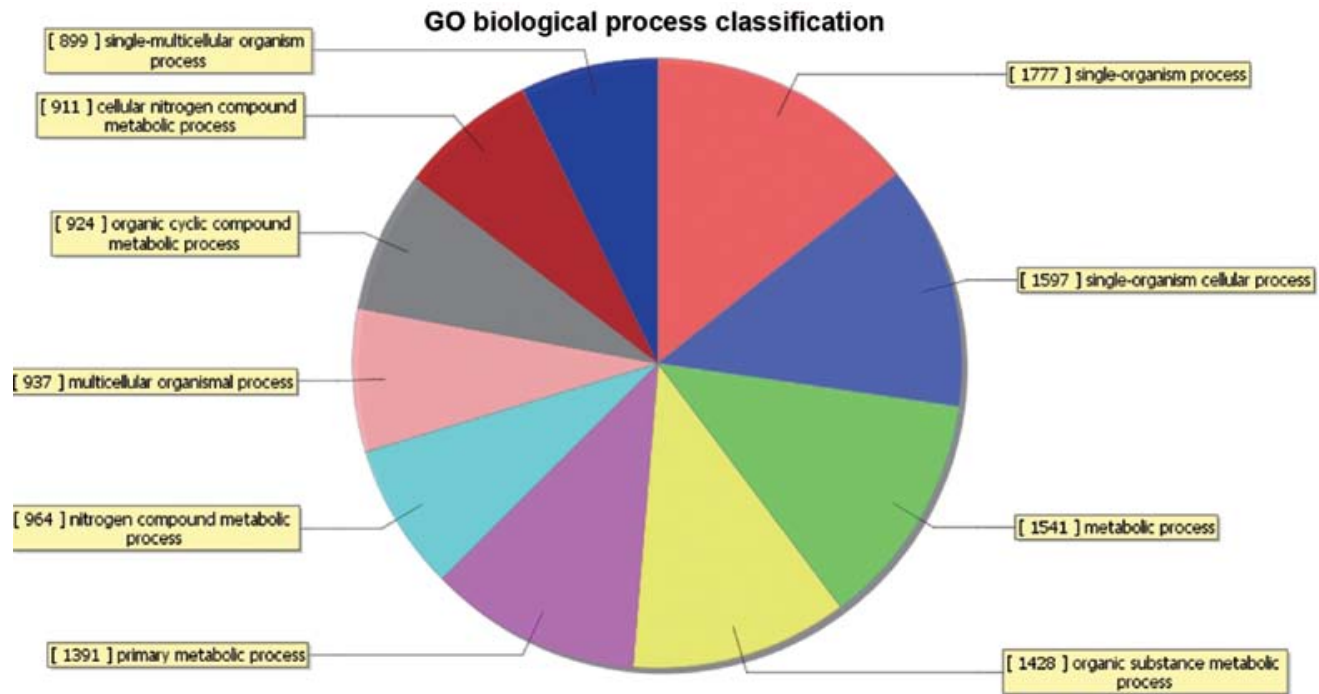

Figure 4. GO biological process classifications of upregulated genes.

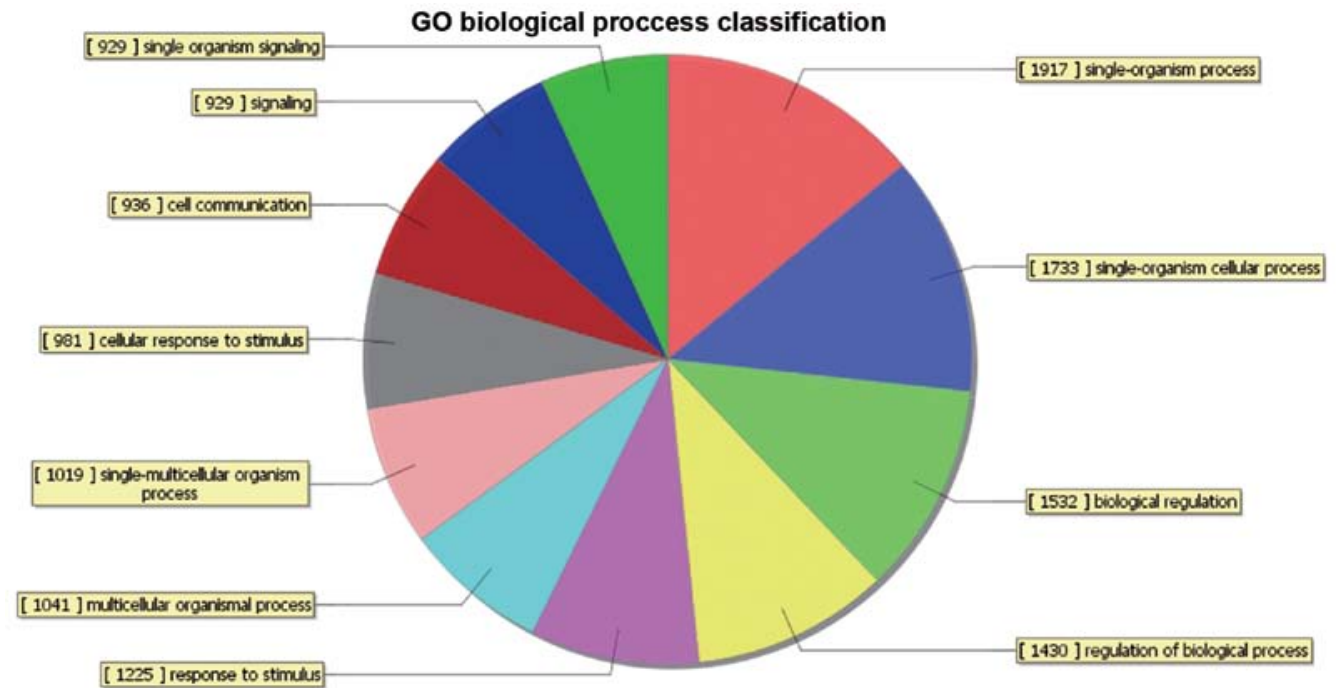

Figure 5. GO biological process classifications of downregulated genes.

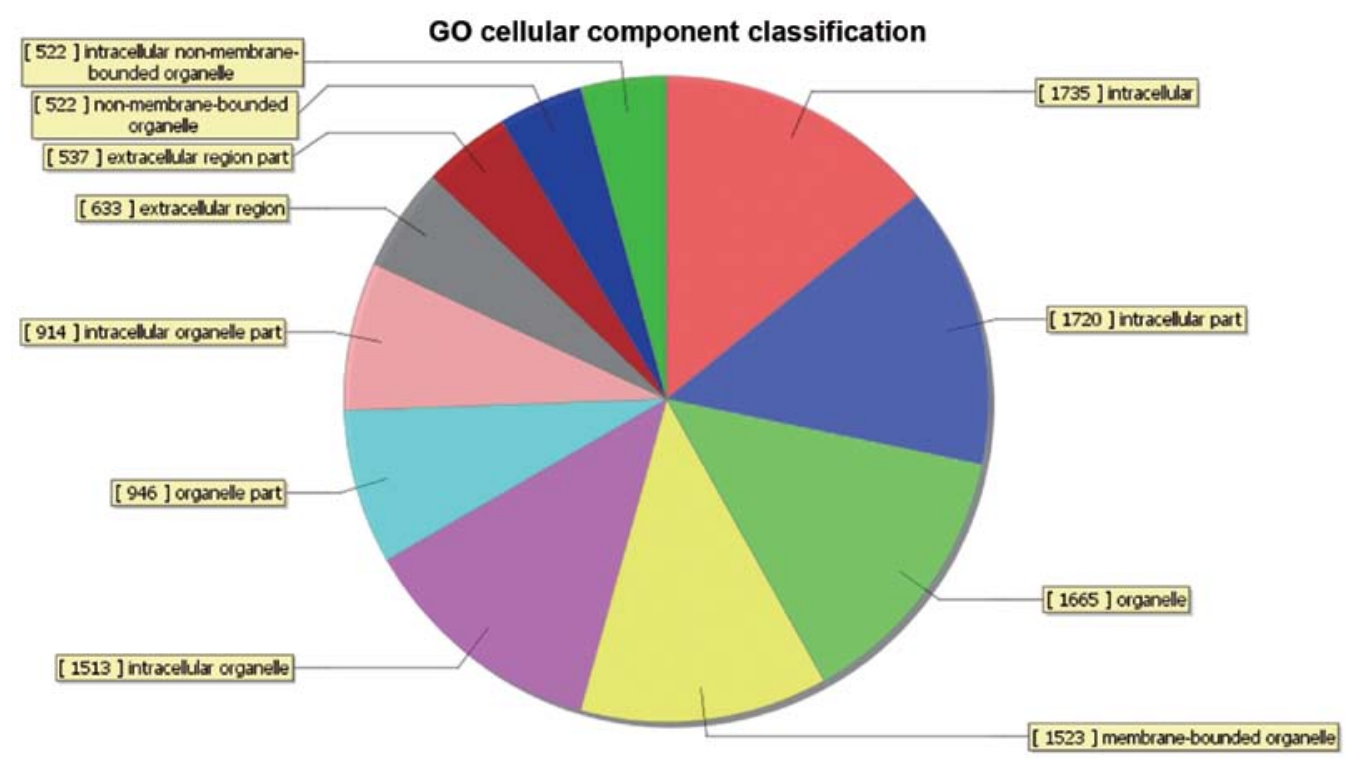

Figure 6. GO cellular component classifications of upregulated genes. 


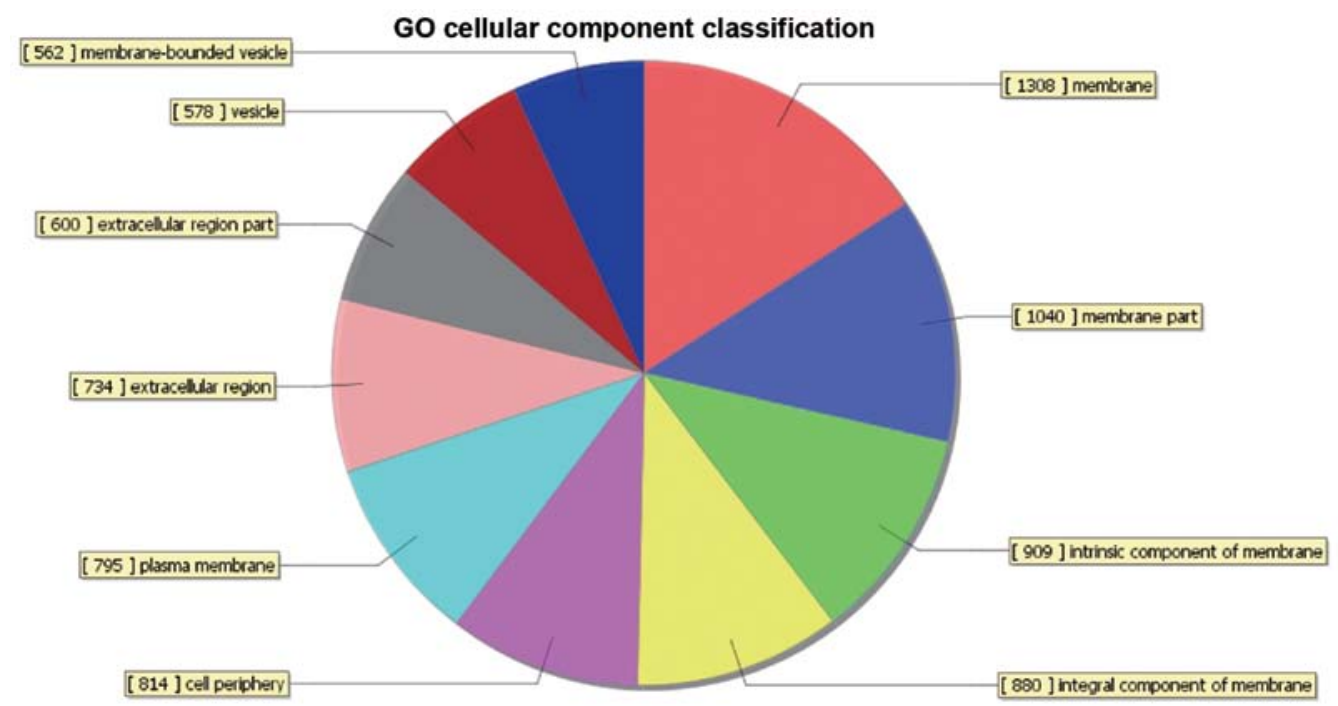

Figure 7. GO cellular component classifications of downregulated genes.

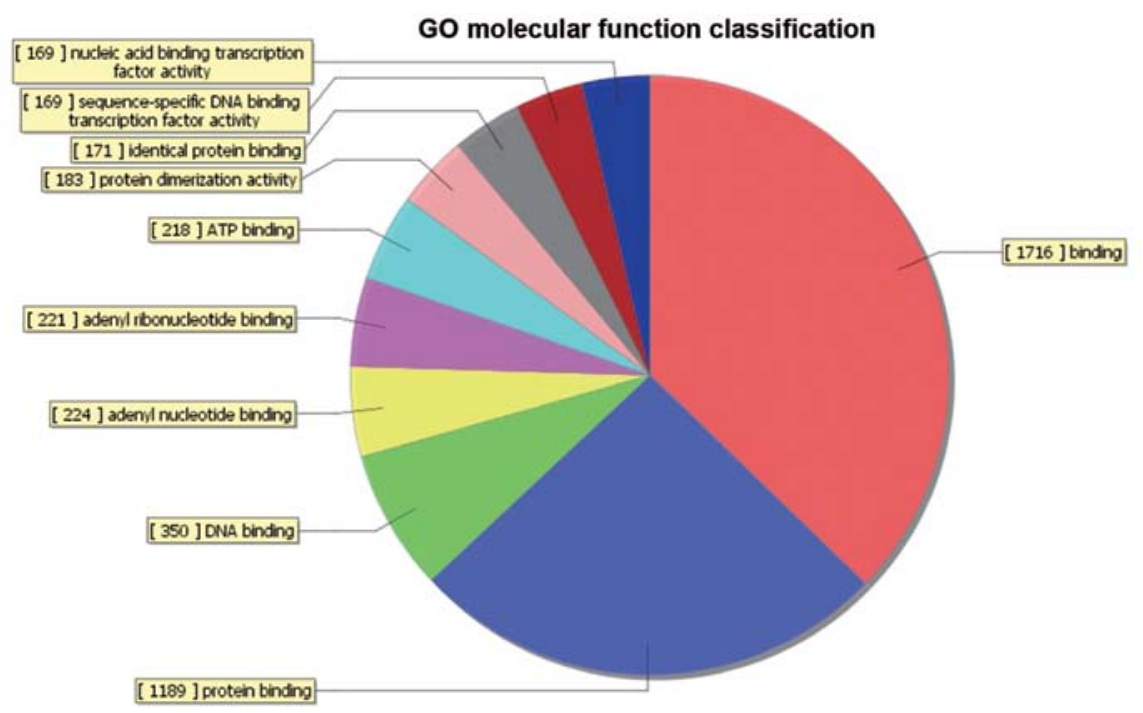

Figure 8. GO molecular function classifications of upregulated genes.

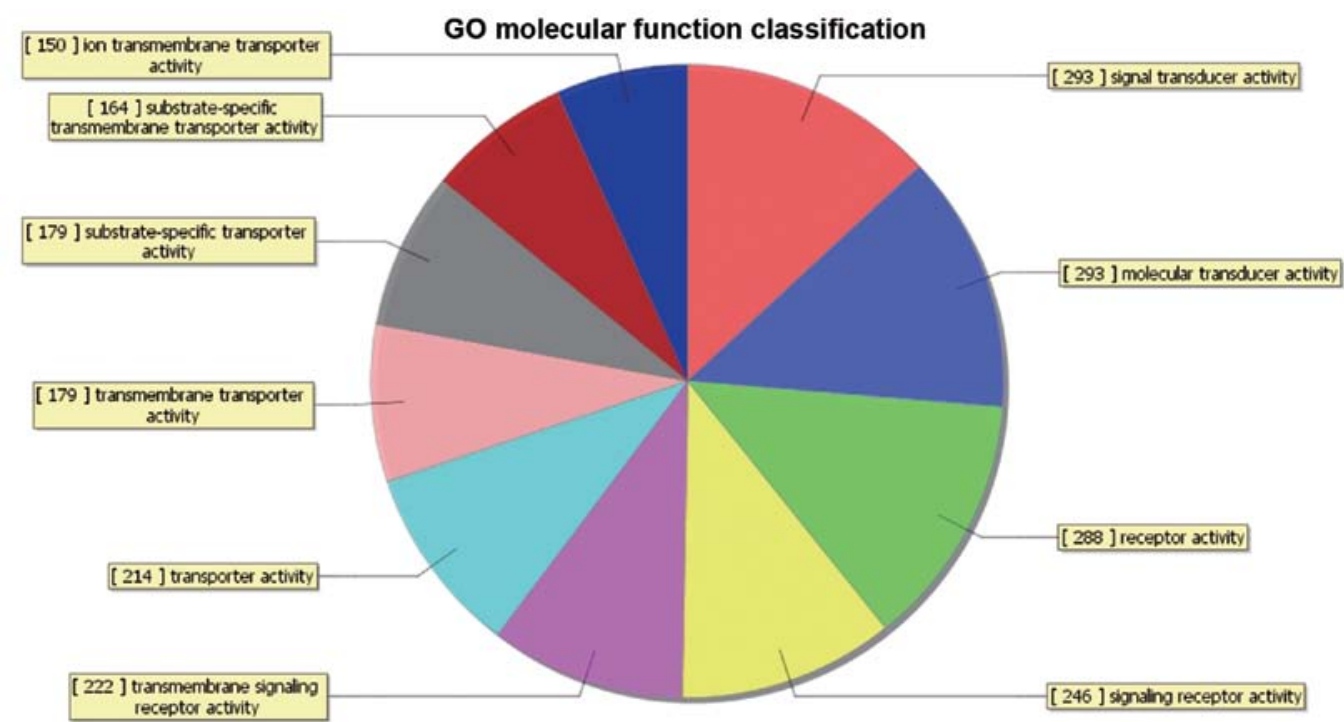

Figure 9. GO molecular function classifications of downregulated genes. 
Table IV. The main pathways.

\begin{tabular}{|c|c|c|c|c|}
\hline Pathway ID & Access information description & Adjust & Count & P-value \\
\hline hsa02010 & $\mathrm{ABC}$ transporters & Upregulated & 44 & $9.85701 \mathrm{E}-06$ \\
\hline hsa03460 & Fanconi anemia pathway & Upregulated & 53 & 0.000519872 \\
\hline hsa05200 & Pathways in cancer & Upregulated & 327 & 0.001215533 \\
\hline hsa03440 & Homologous recombination & Upregulated & 28 & 0.001408952 \\
\hline hsa04974 & Protein digestion and absorption & Upregulated & 88 & 0.002310888 \\
\hline hsa05214 & Glioma & Upregulated & 65 & 0.005344975 \\
\hline hsa05218 & Melanoma & Upregulated & 71 & 0.005613469 \\
\hline hsa04320 & Dorso-ventral axis formation & Upregulated & 24 & 0.006804497 \\
\hline hsa03008 & Ribosome biogenesis in eukaryotes & Upregulated & 85 & 0.007767791 \\
\hline hsa04950 & Maturity onset diabetes of the young & Upregulated & 25 & 0.008931677 \\
\hline hsa05150 & Staphylococcus aureus infection - Homo sapiens & Downregulated & 57 & 5.32523E-06 \\
\hline hsa04978 & Mineral absorption & Downregulated & 51 & 4.16404E-05 \\
\hline hsa04971 & Gastric acid secretion & Downregulated & 75 & 7.6987E-05 \\
\hline hsa00830 & Retinol metabolism & Downregulated & 64 & 0.000143095 \\
\hline hsa04080 & Neuroactive ligand-receptor interaction & Downregulated & 321 & 0.000149431 \\
\hline hsa04514 & Cell adhesion molecules (CAMs) & Downregulated & 145 & 0.000529636 \\
\hline hsa04270 & Vascular smooth muscle contraction & Downregulated & 131 & 0.000677474 \\
\hline hsa04020 & Calcium signaling pathway & Downregulated & 181 & 0.000860266 \\
\hline hsa04672 & Intestinal immune network for IgA production & Downregulated & 49 & 0.000923253 \\
\hline hsa00910 & Nitrogen metabolism & Downregulated & 17 & 0.001285622 \\
\hline
\end{tabular}

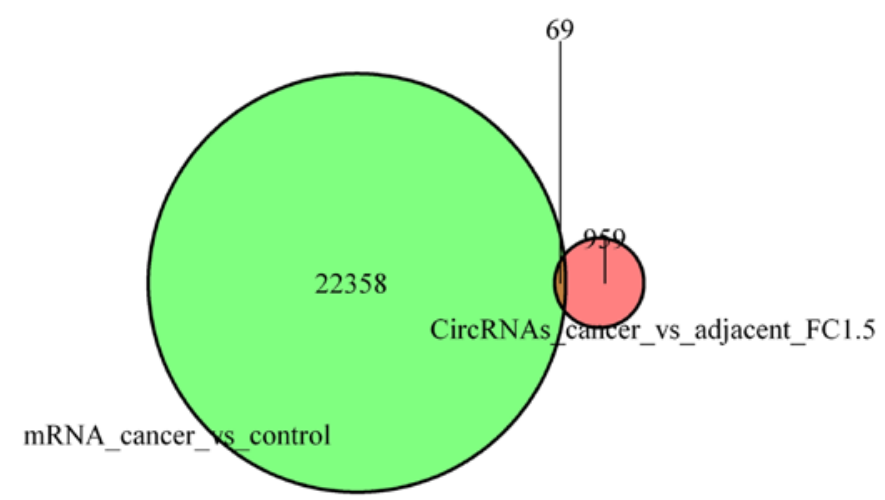

Figure 10. Common genes of related circRNAs-mRNAs.

Analysis of differentially expressed genes in metabolic pathways. In the pathway analysis using the differentially expressed genes, 83 genes were significantly enriched in pathways, from which 20 were selected for the enrichment of gene pathways analysis shown in Table IV.

Correlation analysis of circRNAs and mRNAs in gastric cancer. Because interactions may exist between circRNAs and miRNAs and between miRNAs and mRNAs in gastric cancer tissues and adjacent normal tissues, significantly differentially expressed circRNAs and mRNAs may exist in relationships.

circRNAs with a 1.5-fold difference in expression were associated with the corresponding differential gene expression profile. Only 69 of the differentially expressed circRNA transcripts could be found in the corresponding gene expression profile, as shown in Fig. 10.
A total of 42 genes were upregulated, and significant expression was detected for the following 17 genes $(\mathrm{FC} \geq 2.0$, $\mathrm{P}<0.05)$ : SLAMF6, LAPTM4A, LIN52, MYH9, C16orf72, CD44, MALAT1, RPPH1, SPI1, AXIN1, MLXIP, SLC45A4, Cul4A, RPL18a, ATP13A1, CNPY3 and MSI2. Additionally, 17 genes exhibited downregulated expression, and the following 8 of these genes were significantly expressed (FC $\leq 2.0, \mathrm{P}<0.05)$ : CXXC5, C19orf18, CCS, RABGGTB, ARVCF, GUSBP11, FABP6 and ARIH1.

\section{Discussion}

There is a lack of specific data showing the early stage factors that lead to the high mortality of GC. Therefore, the search for new biological targets for the monitoring and intervention of GC has become an urgent problem.

Differential expression of circRNAs. circRNAs widely exist in many varied types of biological cells (11). They are not easily degraded by RNase and thus, are stably expressed in human cells. Relevant research has shown that circRNA expression levels in human cells may be $>10$-fold greater than the expression levels of their linear isomers $(11,15)$.

Some circRNAs have microRNA response elements. These circRNAs can regulate miRNA expression through chelation, which plays a key role in the regulation of miRNA expression (16). For example, ciRS-7 contains multiple tandem miRNA binding sites and may act as an endogenous miRNA sponge to inhibit the activity of its miR-7 target gene.

Kai et al (17) indicated that the high expression of miR-21-3p might indicate its involvement in the invasion and metastasis of esophageal cancer. In the present study, we 
found differentially expressed circRNAs with corresponding miRNA binding sites. This finding suggests that circRNAs may affect the occurrence and development of gastric cancer and other diseases through interactions with miRNAs.

In the present study, we found 467 differentially expressed circRNAs, of which 214 were significantly upregulated and 253 were significantly downregulated. Most of the differentially expressed circRNAs contained corresponding miRNA binding sites.

Due to the defects in GeneChip technology, great differences exist in screening results. Therefore, in the present study we used qRT-PCR to verify the differential expression of the circRNAs. Since the number of samples was small, we could not confirm whether there was specificity between the selected circRNAs and the GC invasion, metastasis or apoptosis.

To clarify the specific circRNAs associated with GC for early diagnosis and clinical treatment, we need to analyze circRNA expression profiles in a large number of samples. We also need to select more differentially expressed circRNAs to confirm the relationship between their expression levels and $\mathrm{GC}$ at a cellular level in animal experiments. To this end, we may be able to clarify the biological functions of specifically expressed circRNAs in GC.

Differentially expressed genes. Tumors are a type of genetic disease that occur as the result of interactions between multiple genes, leading to their generation and transfer. The deepening of the study of gene functions has furthered our understanding of tumor cell metastasis. On the molecular level, tumor cell metastasis can be divided into the following stages: transformation of normal cells, hyperplasia of tumor cells and tumor cell growth; proliferation; cell cycle survival of tumor cells and extracellular matrix adhesion; release of proteolytic enzymes from tumor cells into the extracellular matrix, resulting in basement membrane hydrolysis; tumor cell infiltration or vascular invasion into organs; escape from host immune mechanisms and the production of new microvessels; and proliferation in the new environment. These processes are related to abnormal expression. Apart from the primary tumor, tumor cells are rarely malignant and most transfers occur during the process of gene exchange. GeneChip technology is a high-throughput method that screens differentially expressed genes through parallel detection; thus, this technology can be used for studies of the mechanisms underlying gastric cancer and metastasis.

Differential expression profiles can be obtained from the Agilent microarray. The chip includes almost all genes found to date. Expression profile microarray data have been used to find new possibilities and gastric cancer-related genes and have provided a wealth of information. Experimental studies on results in cancer tissues and adjacent organizations from the Communist Party of China (CPC) found that 5,460 out of 29,112 mRNA genes exhibited differential expression, including 2,390 genes that were significantly upregulated and 3,070 genes that were significantly downregulated. By analyzing differences in gene clustering, we can more accurately divide gastric cancer tissues from adjacent tissue areas. The comparative expression microarray screened 1,115 upregulated genes and 975 downregulated genes using GO analysis.
The results showed that the upregulated genes were primarily involved in cell metabolism, nucleic acid binding activity of transcription factors, cell generation, DNA binding and other biological processes; these processes may stimulate the cells to lose their normal regulation of growth at the gene level, leading to abnormal hyperplasia and resulting in the formation of gastric cancer. The pathway analysis showed 2,090 genes involved in 83 signaling pathways, including focal adhesion, Rap1 signaling and cytokine receptor interactions, protein digestion and ceiling, alcoholism, transfer RNA and the cell cycle. These pathways also participated in asthma, basal cell carcinoma, bladder and prostate cancer, leishmaniasis, inflammatory bowel disease (IBD), allogeneic shift in the allograft rejection reaction and other metabolic process diseases. In this study, we reported genetic differences in 38 genes involved in the Rap1 signaling pathway. At present, the Rap1 signaling pathway has been associated with cancer based on biological information (18). These findings suggest that mRNA microarray screening of selected differentially expressed genes may be accomplished with a variety of methods to assess the roles involved in the occurrence of gastric cancer.

We performed spectral mining of the mRNA expression data to assess the relevant documents. We found that the differentially expressed genes participated in multiple pathways that were closely related to tumor occurrence. The PDGFRB (platelet-derived growth factor receptor beta polypeptide) gene encodes a platelet derivative growth factor family member and cell surface amino acid kinase receptor. Studies have shown the participation of PDGFR and its ligand in angiogenesis and vascular functions, which suggests that PDGF-B indirectly regulates endothelial cell functions and is an important factor for tumor development $(19,20)$. A research has shown that PDGFRb can selectively regulate and promote colon cancer and osteosarcoma cell growth. Ionization radiation experiments were performed to assess non-small cell lung cancer cell survival and it was found that PDGFR was upregulated in the cell spheroids that survived the radiation exposure (21). Thus, CD44 and PDGFR may be possible markers for non-small cell lung cancer radiotherapy. GREB1 is located in the promoter of the estrogen response element and is a feature of estrogen receptor target genes. Its expression was inversely related to the proto-oncogene epidermal growth factor receptor 2 (HER2) status. In breast cancer cells, GREB1 overexpression promoted cell proliferation and increased the clone formation ability. IL-6/STAT3 regulation by estrogen induced GREB1 transcriptional activity (22). Upregulated expression of the KIT gene, which is a stem cell growth factor receptor and tumor stem cell marker, was associated with gene mutations in a variety of tumors. Akiyama et al (23) noted that the tumor carcinogenic signal c-kit and other tyrosine kinase receptors played very important roles.

In gastric cancer and paracancerous tissues, mRNA expression differences may suggest their diagnostic and treatment values for gastric cancer. This study used expression profile microarray technology to construct gastric cancer and adjacent tissue mRNA expression profiles. These profiles were used to screen differentially expressed mRNAs to identify differentially expressed genes involved in gastric cancer. We assessed signal transduction pathways to provide insights into direction of future studies on the occurrence of gastric cancer 
and to help elucidate the development mechanism to provide an experimental basis.

Relationship between circRNAs and mRNAs. The expression of approximately one-third of human genes is regulated by miRNAs. miRNAs can act through a variety of pathways and can target multiple genes for regulation. A number of miRNAs can also regulate target genes through different pathways (24). An increasing number of studies has shown that miRNAs and mRNAs exist in very complex regulatory relationships. A miRNA regulates multiple target genes that are enriched by one or more signaling pathways. In turn, the miRNA acts on multiple target proteins to regulate signaling pathways to more effectively achieve the miRNA-mRNA regulation network. The ultimate manifestation is the regulation of the cell phenotype and function $(25,26)$. miRNAs also play a regulatory role in disease by interacting with circRNAs. Investigations of these interactions may provide a basis for the elucidation of the disease mechanism, target intervention and clinical diagnostic markers. CDR1as interacts with miR-7 and acts as a miR-7 sponge based on a combination of nearly 70 binding sites, which effectively influence miR-7 target gene activity. CDRlas and miR-7 both exhibit high expression levels in the midbrain development zone. Overexpression in zebrafish embryos results in a fetal brain volume reduction; the midbrain volume can be partially restored by injection of miR-7, suggesting that miR-7 and its interaction with CDR las is at least partially responsible for the biological effect. CDR1as is a cyclic miR-7 inhibitor; thus, CDRlas can influence tumor regulation through miR-7 because miR-7 plays a negative role in regulation. Therefore, CDRlas can fulfill its function as a natural miRNA sponge.

We analyzed circRNA and mRNA expression profiles from gastric cancer tissues and adjacent normal tissues and their correlations. The results showed only 69 differences in circRNA transcripts with corresponding gene expression profile information. For instance, upregulated expression of the tumor metastasis-related 42. MYH9 gene can cause changes in gastric cancer cell invasion and migration (27). CD44 is one type of tumor stem cell marker that is found with high expression in a variety of tumors and contributes to tumor growth, invasion and metastasis (28). MALAT1 [also known as nuclear enrichment of transcription factor 2 (neat2)] is a gene expression regulator of lung cancer metastasis and a prognostic indicator for some solid tumors (29). ARVCF plays a role in renal cell cancer, kidney and prostate cancer. CXXC5 was demonstrated to enhance the ability of ovarian cancer cells to migrate and invade and to inhibit the apoptosis of ovarian cancer cells that occurred during the analysis of its relationship with its associated mRNA (30). We detected differential expression in 69 circRNA transcriptional genes and mRNAs in the gene profiles of the same genes. These results suggest that gene expression is regulated by circRNA and miRNA and miRNA and mRNA interactions in a differential manner. Interactions and mutual influences are likely to have an important impact on the occurrence and development of gastric cancer.

By analyzing circRNAs, mRNAs, and their relationships in gastric cancer tissues and adjacent tissues, we found that circRNAs and mRNAs had a relationship in gastric cancer that led to a more sober understanding: circRNAs are controlled by adsorption and miRNA interactions, thereby establishing a possible role for circRNA-miRNA-mRNA in the regulation of gastric cancer development.

In conclusion, this experiment in eight patients who underwent gastric cancer resection surgery for lesions in gastric cancer tissues and adjacent tissues as the research object used Agilent microarray expression to construct circRNA and mRNA differential expression profiles for the gastric cancer tissues and adjacent tissues. The significant differences in circRNA and mRNA expression in the association analysis suggested that the circRNAs regulated the expression of various genes in gastric cancer. These results contribute to our understanding of the molecular mechanism underlying the development gastric cancer and the search for an effective early diagnostic biomolecule marker for gastric cancer and malignant progression on a theoretical basis. The qRT-PCR validation of differentially expressed circRNAs suggested that RNA and gene expression was regulated in the gastric tissues and adjacent tissues.

To summarize, the present study draws the following conclusions: i) differential expression of circRNAs and the corresponding miRNAs interact through circRNA binding sites to regulate the expression of target genes. These circRNAs are likely to become new molecular biomarkers for gastric cancer in the future. ii) Genes involved in cell metabolism, nucleic acid binding activity of transcription factors, the generation of organelles and DNA binding (biological processes) may be prompting cells to lose their normal growth regulation at the gene level. These genes are also involved in the Rap1 signaling pathway, cell factor interactions, protein digestion and absorption, basal cell carcinoma, prostate cancer, the allogeneic shift in the allograft rejection reaction and other metabolic process diseases. The genes may be differentially expressed through a variety of pathways and play roles in the occurrence of gastric cancer. iii) CD44, CXXC5, MYH9, MALAT1 and other genes are regulated through interactions between circRNAs and miRNAs and between miRNAs and mRNAs via different mechanisms, including direct interactions and mutual influence. These interactions may be important influences on the occurrence and development of gastric cancer.

\section{References}

1. Jemal A, Bray F, Center MM, Ferlay J, Ward E and Forman D: Global cancer statistics. CA Cancer J Clin 61: 69-90, 2011.

2. Park DJ, Thomas NJ, Yoon C and Yoon SS: Vascular endothelial growth factor a inhibition in gastric cancer. Gastric Cancer 18: 33-42, 2015.

3. Karimi P, Islami F, Anandasabapathy S, Freedman ND and Kamangar F: Gastric cancer: Descriptive epidemiology, risk factors, screening, and prevention. Cancer Epidemiol Biomarkers Prev 23: 700-713, 2014.

4. Degiuli M and Calvo F: Survival of early gastric cancer in a specialized European center. Which lymphadenectomy is necessary? World J Surg 30: 2193-2203, 2006.

5. Schwarz RE and Smith DD: Clinical impact of lymphadenectomy extent in resectable gastric cancer of advanced stage. Ann Surg Oncol 14: 317-328, 2007.

6. Wilusz JE and Sharp PA: Molecular biology. A circuitous route to noncoding RNA. Science 340: 440-441, 2013.

7. Hansen TB, Jensen TI, Clausen BH, Bramsen JB, Finsen B, Damgaard CK and Kjems J: Natural RNA circles function as efficient microRNA sponges. Nature 495: 384-388, 2013.

8. Maass PG, Luft FC and Bähring S: Long non-coding RNA in health and disease. J Mol Med (Berl) 92: 337-346, 2014. 
9. Valdmanis PN and Kay MA: The expanding repertoire of circular RNAs. Mol Ther 21: 1112-1114, 2013.

10. Burd CE, Jeck WR, Liu Y, Sanoff HK, Wang Z and Sharpless NE: Expression of linear and novel circular forms of an INK4/ ARF-associated non-coding RNA correlates with atherosclerosis risk. PLoS Genet 6: e1001233, 2010.

11. Jeck WR, Sorrentino JA, Wang K, Slevin MK, Burd CE, Liu J, Marzluff WF and Sharpless NE: Circular RNAs are abundant, conserved, and associated with ALU repeats. RNA 19: 141-157, 2013.

12. Li Z, Huang C, Bao C, Chen L, Lin M, Wang X, Zhong G, Yu B, $\mathrm{Hu}$ W, Dai L, et al: Exon-intron circular RNAs regulate transcription in the nucleus. Nat Struct Mol Biol 22: 256-264, 2015.

13. Li F, Zhang L, Li W, Deng J, Zheng J, An M, Lu J and Zhou Y: Circular RNA ITCH has inhibitory effect on ESCC by suppressing the Wnt/ $\beta$-catenin pathway. Oncotarget 6: 6001-6013, 2015.

14. Dai Y, Sui W, Lan H, Yan Q, Huang H and Huang Y: Comprehensive analysis of microRNA expression patterns in renal biopsies of lupus nephritis patients. Rheumatol Int 29: 749-754 2009.

15. Memczak S, Jens M, Elefsinioti A, Torti F, Krueger J, Rybak A, Maier L, Mackowiak SD, Gregersen LH, Munschauer M, et al: Circular RNAs are a large class of animal RNAs with regulatory potency. Nature 495: 333-338, 2013.

16. Salzman J, Gawad C, Wang PL, Lacayo N and Brown PO Circular RNAs are the predominant transcript isoform from hundreds of human genes in diverse cell types. PLoS One 7: e30733, 2012.

17. Ghosal S, Das S, Sen R, Basak P and Chakrabarti J: Circ2Traits A comprehensive database for circular RNA potentially associated with disease and traits. Front Genet 4: 283, 2013.

18. Doberstein K, Bretz NP, Schirmer U, Fiegl H, Blaheta R, Breunig C, Müller-Holzner E, Reimer D, Zeimet AG and Altevogt P: miR-21-3p is a positive regulator of L1CAM in several human carcinomas. Cancer Lett 354: 455-466, 2014.

19. Fukuhara S, Sakurai A, Sano H, Yamagishi A, Somekawa S, Takakura N, Saito Y, Kangawa K and Mochizuki N: Cyclic AMP potentiates vascular endothelial cadherin-mediated cell-cell contact to enhance endothelial barrier function through an Epac-Rap1 signaling pathway. Mol Cell Biol 25: 136-146, 2005 .
20. Arai K, Sakamoto R, Kubota D and Kondo T: Proteomic approach toward molecular backgrounds of drug resistance of osteosarcoma cells in spheroid culture system. Proteomics 13: 2351-2360, 2013

21. Cho HJ, Baek KE, Kim IK, Park SM, Choi YL, Nam IK, Park SH, Im MJ, Yoo JM, Ryu KJ, et al: Proteomics-based strategy to delineate the molecular mechanisms of RhoGDI2-induced metastasis and drug resistance in gastric cancer. J Proteome Res 11: 2355-2364, 2012

22. PLoS One Editors: Retraction: GREB1 functions as a growth promoter and is modulated by IL6/STAT3 in breast cancer. PLoS One 9: e102287, 2014.

23. Akiyama H, Watanabe T, Wakabayashi K, Nakade S, Yasui S, Sakata K, Chiba R, Spiegelhalter F, Hino A and Maitani T: Quantitative detection system for maize sample containing combined-trait genetically modified maize. Anal Chem 77: 7421-7428, 2005.

24. Feigin VL and Findlay M: Advances in subarachnoid hemorrhage. Stroke 37: 305-308, 2006.

25. van der Voet M, Olson JM, Kuivaniemi H, Dudek DM, Skunca M, Ronkainen A, Niemelä M, Jääskeläinen J, Hernesniemi J, Helin K, et al: Intracranial aneurysms in Finnish families: Confirmation of linkage and refinement of the interval to chromosome 19q13.3. Am J Hum Genet 74: 564-571, 2004.

26. Juvela S: Natural history of unruptured intracranial aneurysms: Risks for aneurysm formation, growth, and rupture. Acta Neurochir Suppl 82: 27-30, 2002.

27. Liang S, He L, Zhao X, Miao Y, Gu Y, Guo C, Xue Z, Dou W, $\mathrm{Hu} \mathrm{F}$, Wu K, et al: MicroRNA let-7f inhibits tumor invasion and metastasis by targeting MYH9 in human gastric cancer. PLoS One 6: e18409, 2011.

28. Naor D, Nedvetzki S, Golan I, Melnik L and Faitelson Y: CD44 in cancer. Crit Rev Clin Lab Sci 39: 527-579, 2002

29. Gutschner T, Hämmerle M and Diederichs S: MALAT1 - a paradigm for long noncoding RNA function in cancer. $\mathrm{J}$ Mol Med (Berl) 91: 791-801, 2013.

30. Wang JH, Ren Y, Zhang R, Han Y, Sheng YH, Hou WJ and Ao HF: Effects of CXXC finger protein 5 up-regulated expression in epithelial ovarian cancer. China Oncol 25: 260-268, 2015 (In Chinese). http://www.china-oncology.com/EN/Y2015/V25/ I4/260. 